ESJ Social Sciences

\title{
Contribution du capital humain et du renforcement des capacites a la croissance economique des pays de l'afrique subsaharienne : une approche par la technologies de frontiere
}

\author{
Mamadou Coulibaly, Docteur \\ Ecole Normale Supérieur d'Abidjan, Côte d'Ivoire \\ Jacques Konan Konan, Docteur \\ LAMP, Université Alassane Ouattara, Côte d'Ivoire
}

Doi:10.19044/esj.2021.v17n5p131

Submitted: 21 August 2020

Accepted: 20 January 2021

Published: 28 February 2021
Copyright 2021 Author(s)

Under Creative Commons BY-NC-ND

4.0 OPEN ACCESS

Cite As:

Coulibaly M. \& Konan K.J. (2021). Contribution du capital humain et du renforcement des capacites a la croissance economique des pays de l'afrique subsaharienne : une approche par la technologies de frontiere. European Scientific Journal, ESJ, 17(5), 131.

https://doi.org/10.19044/esj.2021.v17n5p131

\section{Résumé}

La politique du renforcement des capacités et l'amélioration du système éducatif semblent être contributifs à la croissance économique. Mais, l'objectif d'un rapport étroit entre éducation et accumulation de richesse reste loin d'être atteint. Ainsi, la prise en compte de la technologie de frontière semble être importante dans l'analyse relationnelle entre capital humain et croissance économique. La présente analyse se penche sur la contribution du capital humain et du renforcement des capacités à la performance économique des pays d'Afrique subsaharienne en tenant compte de la technologie de frontière. Les résultats empiriques avec les données de panel par la méthode des moments généralisés ressortent que les pays africains sont loin de la technologie de frontière du monde. Aussi, les capacités renforcées et de l'éducation n'ont-elles aucun effet significatif sur le PIB par tête de ces pays. Le niveau de la technologie de frontière des pays africains améliore l'influence de l'éducation secondaire sur le PIB par tête de ces pays de 33,40\%. Finalement, La technologie de frontière n'a aucun apport contributif de l'incidence des capacités sur la croissance du PIB par tête des pays d'Afrique Subsaharienne. 
Mots clés : Capital humain, technologie de frontière, croissance économique, capacités, Afrique Subsaharienne

\title{
Contribution of Human Capital and Capacity Building to The Economic Growth of Sub-Saharan African Countries: A Frontier Technology Approach
}

\author{
Mamadou Coulibaly, Docteur \\ Ecole Normale Supérieur d'Abidjan, Côte d'Ivoire \\ Jacques Konan Konan, Docteur \\ LAMP, Université Alassane Ouattara, Côte d'Ivoire
}

\begin{abstract}
The policy of capacity building and improvement of the education system seem to be contributing to economic growth. But the goal of a close relationship between education and wealth accumulation remains far from being achieved. Thus, the consideration of frontier technology seems to be important in the relational analysis between human capital and economic growth. This analysis examines the contribution of human capital and capacity building to the economic performance of sub-Saharan African countries taking into account frontier technology. Empirical results with panel data by the generalized moments method show that African countries are far from the world's frontier technology. Also, enhanced capacities and education have no significant effect on countries' per capita GDP. The level of frontier technology of African countries improves the influence of secondary education on the per capita GDP of these countries by $33.40 \%$. Finally, Frontier Technology does not contribute to the impact of capabilities on per capita GDP growth in Sub-Saharan African countries.
\end{abstract}

Keywords: Human capital, frontier technology, economic growth, capability building, Sub-Saharan-Africa

\section{Introduction}

La performance économique d'un pays est fonction de sa capacité à atteindre ses objectifs fixés. L'atteinte de ces objectifs dépend du niveau de dotation de certains indicateurs économiques comme le capital humain. Comme soulignés par Hall et Jones (1999) et Pritchett (2000), les institutions encouragent l'accumulation du capital humain et l'amélioration du PIB. Le capital humain représente $22 \%$ de la croissance de la productivité des pays d'OCDE (Angel et Antonio, 2002). Un corpus d'étude a exhibé sa part 
importante pour la croissance économique. Schultz (1972) et Denison (1962) reconnaissent que, le capital humain a une forte contribution pour la croissance économique. Mais l'évidence d'un rapport étroit entre l'éducation et l'accumulation de richesse reste loin d'être atteinte. Selon Pritchett (2000), Bils et Klenow (2000) et Benhabits et Soiegel (1994), le lien entre le capital humain et la croissance est inexistant, ou très faible et lorsqu'il existe, l'éducation agit négativement sur le revenu de façon significative.

Selon Aghion et Cohen (2004), limiter la mesure du rendement de l'éducation par une année supplémentaire, c'est la sous-estimer, car certaines externalités ne sont pas prises en compte pour son évaluation. Aghion et al $(2005 ; 2008)$ et Acemoglu et al (2006) s'évertuent à montrer que ce lien dépend des composantes du capital humain et du niveau de la technologie des pays par l'adaptation ou l'innovation. Cependant les activités d'innovation et d'imitation dépendent de la main d'œuvre qualifiée. La nécessité de ces deux activités pour la performance économique est fonction de la situation de proximité du pays à la frontière technologique. En dehors de la part contributive du capital humain à la santé économique, l'apport d'une amélioration de l'effet du capital humain sur la croissance aurait une de ses sources dans la politique du renforcement des capacités. Cette politique permet aux pays de décider de leurs priorités et de prendre l'initiative pour relever les défis du développement (Mahshi, 2006). Concernant la santé économique africaine, le niveau du capital humain est insignifiant pour répondre aux défis de développement. Sur cette question africaine, Acemoglu et Robinson (2010) trouvent que l'Afrique dispose d'une abondance de main d'œuvre non qualifiée source de défaillance économique.

$\mathrm{Au} \mathrm{vu}$ de ces observations, se pose la question suivante : L'amélioration des capacités et des composantes du capital humain permetelle une bonne activité d'adaptation de la technologie des pays de l'Afrique subsaharienne ? L'objectif de cette étude consiste à déterminer l'influence du capital humain et celle des capacités renforcées sur la croissance économique des pays de l'Afrique subsaharienne en tenant compte de leur position par rapport à la technologie de frontière du monde. Cette étude est structurée en deux sections : (ii) la revue de littérature portant sur les différents concepts et (iii) l'approche méthodologique.

\section{2- Capacités, capital humain et progrès technique pour la croissance économique}

La contribution du capital humain et celle des capacités renforcées à la croissance économique des pays font objet de plusieurs analyses théoriques et empiriques. Ces analyses permettent de comprendre les différents fondements des concepts et leurs apports à la santé économique des pays. 


\subsection{Approche économique des capacités}

L'origine de la théorie des capacités a pour germe celle de la capabilité d'Amartya Sen. La capabilité de Sen est la liberté qu'un individu a pour choisir le type de vie qu'il souhaite mener (Reboud, 2008). Baser et Morgan (2008) étendent cette conception de Sen à cinq capabilités centrales dont la combinaison de l'ensemble assure la capacité globale qui permet de quitter un état A vers l'obtention d'un autre B amélioré. L'ensemble des cinq capabilités centrales renferment la capabilité centrale de s'engager et d'exécuter, de soutenir des techniques et tâches logistiques, d'attirer les ressources, de s'adapter et s'auto-qualifier et enfin d'assurer la cohérence. Selon Morgan (2006), Engel et al (2007), Baser et Morgan (2008) et Ubel et al (2010), la combinaison des cinq capabilités entrâne la capacité globale qui crée de la valeur pour le développement économique. C'est l'aptitude pour un individu ou une société à fixer les objectifs de développement et à les atteindre avec succès, la renforcer devient un processus (ACBF, 2011).

Le processus des capacités a une incitation importante aux activités économiques, c'est pourquoi Ksafack (2011) précise que la capacité est une connaissance acquise, permettant à une personne de réussir dans l'exercice d'une activité physique ou intellectuelle. La renforcer revient à développer la compétence humaine, individuelle ou collective pour une croissance soutenable. Ce processus est inévitable pour les pays en développement du moment qu'il consolide la prospérité économique, la stabilité politique et la justice sociale pour tous les citoyens, ainsi que la mobilisation des ressources internes au solde de l'investissement local, (ACBF, 2015). Par conséquent, il existe cinq (5) étapes ${ }^{1}$. La première est d'obtenir l'engagement des parties prenantes au renforcement des capacités. La deuxième consiste à examiner les capacités existantes et les manquantes. La troisième étape porte sur la formulation de stratégies de renforcement des capacités. La quatrième étape est relative à la mise en œuvre des stratégies de renforcement des capacités. Quant à la dernière étape, elle porte sur l'Evaluation du renforcement des capacités.

Le processus est fondamental pour le secteur public, privé et les ONG. Il existe au niveau micro comme macro. L'apport micro de capacités concerne la compétence du personnel d'une organisation. Au niveau macro pour ce qui est de l'administration publique c'est la capacité d'augmenter le bien-être des citoyens, en fournissant des services appropriés, facilitant la santé, la création d'emplois, l'éducation etc. C'est le niveau soutenable du rendement public du fait qu'il avantage les pays à contrôler leurs affaires avec succès (Morgan, 2006). North et al (1999) étendent la conception à travers la théorie de l'ordre

${ }^{1}$ Les cinq étapes du processus du renforcement de capacité sont inspirées de PNUD (2008), OCDE (2006) et des rapports d'ACBF. 
public qui implique le renforcement de la capacité de l'Etat comme la création des responsabilités crédibles, des droits de propriétés solides source de l'économie du marché.

\section{2-2 Lien entre capital humain, progrès technique et croissance économique}

La relation éducation et progrès technique, fait l'objet d'un apport contributif à l'explication des limites de l'efficacité du capital humain à la seule notion de l'éducation pour la croissance économique. Romer (1991) ressort trois notions essentielles de celui-ci, à savoir : le progrès technique améliore l'accumulation du capital et leur combinaison est source de la productivité horaire. De plus, il est une décision volontaire des individus pour répondre aux exigences du marché. En sus, les modalités d'utilisation du progrès technique diffèrent de celles des autres biens.

Selon Romer (1990), un ingénieur à la recherche aujourd'hui a le même capital humain qu'un autre d'un siècle passé. Mais celui d'aujourd'hui est plus efficace grâce à l'acquisition des connaissances accumulées pendant les 100 ans passés. En effet Gerschenkron (1962) écrit que les économies relativement en arrière au $19^{\text {iem }}$ siècle telles que, l'Allemagne, la France et la Russie ont pu rattraper rapidement les plus avancées en entreprenant de grands investissements pour adopter des technologies de frontière. Celle-ci est grandement étayée par Berthélemy et al (1997), pour eux un niveau d'éducation élevé renforce la capacité d'un pays à imiter et à utiliser les technologies découvertes dans les pays avancés. L'analyse de Vandenbussche et al (2006), montre que la main d'œuvre qualifiée influence plus la croissance lorsque le pays est proche de la frontière technologique et le capital humain non qualifié est convenable aux activités d'imitation pour la croissance économique du pays. Cette thèse est soutenue par Islam (2010), pour qui le capital humain influence plus la croissance économique à l'approche de la technologie de frontière pour les pays pauvres comme riches.

\section{2-3 Revue empirique du capital humain et sa relation avec la technologie à la croissance économique}

Les réflexions de Hanushek et Kimko (2000) sur l'apport du capital humain à la croissance économique des pays est plus orienté sur les niveaux d'influence distincte, c'est-à-dire une année d'étude ' d'un pays i'" n'a pas les mêmes influences qu'une année d'étude dans "un autre pays j'". Leur étude portant sur 36 pays prouve que, contrairement à l'indicateur quantitatif, l'indicateur qualitatif de l'éducation a un effet très significatif et positif sur la croissance économique. Dans la même logique, Barro (2001) montre par la méthode des triples moindres carrés que, la qualité de l'éducation a une plus forte influence que la quantité mesurée par les niveaux moyens d'achèvement 
des études secondaires et supérieures dont l'impact sur la croissance est nul. Altinock (2007) en élargissant l'échantillon à 105 pays, aboutit au même résultat.

Pour déterminer l'apport de certains indicateurs de la croissance de long terme, Banerjee et Sinha (2014) utilisent l'estimateur des MCO et trouvent que le renforcement des capacités locales et la technologie venant d'ailleurs déterminent largement la croissance de 1950 à 2010 en Inde. Avec la méthode des MMG, Ang et al (2011) trouvent à nouveau sur l'ensemble de l'échantillon que l'éducation primaire et secondaire a une plus forte dynamique sur la croissance que l'enseignement supérieur.

L'étude de Islam (2010) confirme l'accroissement de l'effet du capital humain (éducation supérieure) sur la croissance des pays à revenu moyen et élevé, cet effet augmente à mesure que, ces pays se rapprochent de la technologie du monde. A l'approche de la technologie mondiale, la contribution de la main d'œuvre qualifiée (éducation supérieure) masculine est plus importante que la féminine pour les pays développés. Sur un panel de 20 pays de l'OCDE, Aghion et Cohen (2004) étendent la décomposition du capital humain regroupée en deux niveaux. Le premier regroupe les niveaux primaire, secondaire et Baccalauréat plus deux $(+2)$ et l'autre, le niveau supérieur. Les résultats indiquent qu'à un seuil de $24 \%$ de la frontière technologique, tout pays en deçà de ce seuil doit investir dans le premier groupe et au-delà il faut privilégier l'investissement dans le cycle supérieur.

\section{Approche méthodologique}

Pour cette partie empirique, la spécification du modèle pour permettre d'analyser l'apport des composantes du capital humain et les capacités renforcées sur la croissance économique à travers la technologie de frontière s'impose. Ainsi, la spécification proposée par Vandenbussche et al (2006) et celle de Ang et al (2011) sont la source de spécification du modèle de cette étude. Mais, c'est la mesure de la technologie de frontière préconisée par Acemoglu et al (2006) qui est utilisée dans cette étude empirique. Ainsi la spécification du modèle est présentée comme :

$$
g_{i, t(2011-2016)}=\alpha_{0}+\alpha_{1} \ln \left(\frac{y_{i, t}}{y_{U S, t}}\right)+\alpha_{2} f_{i, t}+\alpha_{3} f_{i, t} * \ln \left(\frac{y_{i, t}}{y_{U S, t}}\right)+\beta X_{i, t}+\varepsilon_{i, t}
$$

Avec $g_{i, t(2011-2016)}$ le taux de croissance du PIB/tête du pays $i$ entre 2011 et 2016, $y_{i, t}$ et $y_{U S, t}$ représentant respectivement le niveau du PIB par habitant du pays i et celui des Etats-Unis à la période $t \cdot \frac{y_{i, t}}{y_{U S, t}}$ désigne le niveau de la proximité de la technologie de frontière du pays i à la date $t$, mesuré par le PIB par tête du pays i sur le PIB par tête des Etats-Unis. Le choix des Etats-Unis est justifié 
par Islam (2010) sur la base d'être le leader de la technologie ainsi que sa place majoritaire de partenaire commercial des pays du monde. $f_{i, t}$ est la partie de la population ayant un niveau de capital humain ou un niveau de capacités renforcées. Le produit $f_{i, t} * \ln \left(\frac{y_{i, t}}{y_{U S, t}}\right)$, représente l'effet d'interaction entre l'indicateur des capacités (ICA) ou le capital humain et la technologie de frontière.

$X_{i, t}$ est la matrice des variables de contrôle (le degré d'ouverture, le taux d'inflation, le flux de l'investissement direct étranger) retenues pour cette étude. La localisation géographique 2 et le développement financier retenus par Ang et al (2011) ne sont pas intégrés. Avec Aghion et Cohen (2004), la technologie de frontière est telle que : $a_{i, t}=\frac{y_{i, t}}{y_{U S, t}}$, lorsque $a_{i, t}$ est proche de zéro (0), cela suppose que le pays est loin de la technologie de frontière et lorsque $a_{i, t}$ est proche de un (1), le pays est proche de la frontière technologique. Les deux équations de l'étude à estimer sont :

$$
\begin{aligned}
& g_{i, t(2011-2016)}=\alpha_{0}+\alpha_{1}\left(\frac{y_{i, t}}{y_{U S, t}}\right)+\alpha_{2} H_{i, t}+\alpha_{3} H_{i, t} *\left(\frac{y_{i, t}}{y_{U S, t}}\right)+\beta_{1} D O_{i, t}+\beta_{2} i n f_{i, t}+\beta_{3} f I D E_{i, t}+\varepsilon_{i, t}(2) \\
& g_{i, t(2011-2016)}=\alpha_{0}+\alpha_{1}\left(\frac{y_{i, t}}{y_{U S, t}}\right)+\alpha_{2} C A_{i, t}+\alpha_{3} C A_{i, t} *\left(\frac{y_{i, t}}{y_{U S, t}}\right)+\beta_{1} D O_{i, t}+\beta_{2} i n f_{i, t}+\beta_{3} f I D E_{i, t}+\varepsilon_{i, t}(3)
\end{aligned}
$$

L'équation 2 exprime la contribution du capital humain à la croissance économique à travers la technologie de frontière et l'équation 3 concerne l'apport des capacités renforcées à la croissance économique en tenant compte de la technologie de frontière. Avec $D O$ le degré d'ouverture, inf le taux d'inflation et $f I D E$ le taux d'IDE qui est égal au rapport entre l'IDE et le PIB. A l'exception des capacités renforcées3, les autres indicateurs proviennent de la base des données de la banque mondiale. Pour ce qui est des capacités, selon les différents rapports de l'ACBF, l'indice ICA est la moyenne harmonique des quatre indicateurs de classe (environnement institutionnel, le processus de mise en œuvre, les résultats de développement au niveau national et les résultats en matière de renforcement des capacités). Chacun des indicateurs est agrégé d'une évaluation quantitative et qualitative de diverses

${ }^{2}$ Les données pour la localisation géographique fournies par Landlocked Economies data base des pays d'Afrique subsaharienne ne sont pas disponibles

3 Les données sur les capacités renforcées mesurées par Indice de Capacité en Afrique (ICA), proviennent des différents rapports sur les capacités en Afrique qui sont fournis par la Fondation pour le Renforcement des Capacités en Afrique. L'ICA varie de 0 à 100 et permet aux pays d'être classés en cinq (5) catégories de niveau de capacité : de zéro (0) à vingt (20) niveau très bas, de vingt (20) à quarante (40) niveau bas, de quarante (40) à soixante (60) niveau moyen, de soixante (60) à quatre-vingt 80 niveau élevé et de quatre-vingts (80) à cent (100) niveau très élevé. 
composantes ${ }^{4}$. Avec les données de panel dont la période part de 2011 à 2016, l'effet direct et indirect du capital humain ainsi que les capacités renforcées à travers la technologie de frontière sur la croissance économique peut être analysé.

\section{Résultats de l'étude}

Il est important de passer à l'analyse du comportement naturel des données et par la suite à l'analyse empirique des résultats du modèle. Ainsi, le tableau I ci-dessous désigne la statistique descriptible des variables et le tableau II renferme les différents résultats obtenus du modèle de l'étude.

Tableau I : Statistique descriptive des variables utilisées

\begin{tabular}{llllll}
\hline Variables & $\begin{array}{l}\text { Nombres } \\
\text { d'observat }\end{array}$ & Moyenne & Ecart Type & Minimu & Maximu \\
\hline CroPIB/tt & 200 & 1.88 & 4,38 & $-36,82$ & 17,99 \\
ICA & 200 & 42,61 & 13,67 & 10,02 & 70,8 \\
Techn_fr & 200 & 0,035 & 0,044 & 0,005 & 0,022 \\
R_E_E_E & 153 & 25,61 & 10,68 & 9,82 & 68,13 \\
DO & 240 & 0,35 & 0,38 & 0,01 & 3,55 \\
Tx_IDE & 240 & 0,06 & 0,10 & $-0,05$ & 0,85 \\
Inflation & 237 & 6,10 & 6,18 & $-3,70$ & 37,14 \\
Ratio_IDE & 170 & 2,8147 & 0,5879 & 1,5 & 4 \\
Inflation & 196 & 5,7766 & 5,5220 & $-3,7042$ & 33,2242 \\
Taux Epar & 186 & 0,1317 & 0,21672 & $-0,8142$ & 0,9592 \\
\hline
\end{tabular}

Source : Auteur, réalisé à partir des données de l'ACBF et banque mondiale

Les résultats du tableau confirment que la croissance du PIB par tête en moyenne est de $1,88 \%$ avec un niveau de la technologie de frontière de 0,035. Selon l'analyse de Aghion et al (2004), ce niveau de technologie situe ces pays loin de la frontière technologique du monde. Le niveau des capacités avec une valeur de 42,61 situe les pays africains dans la catégorie du niveau moyen des capacités. Comme la plupart des travaux empiriques, il est impossible d'exclure les problèmes d'endogénéité qu'il sied de résoudre ou de réduire. Selon Wooldridge (2004), il existe trois principales sources d'endogénéité : la simultanéité ou la causalité inverse, omission et erreur de mesure des variables explicatives pertinentes. Cette question de biais d'endogénéité, justifie le choix de la méthode des moments généralisés (MMG) qui repose sur les hypothèses suivantes :

- $H_{0}$ : absence de corrélation d'ordre 2 des résidus ;

- $H_{1}$ : présence de corrélation d'ordre 1 des résidus.

4 La description des variables des capacités et leurs calculs sont purement les produits des différents rapports de l'ACBF. 
Les différents résultats de l'estimation du modèle incluant les capacités, la qualité du capital humain et la technologie des frontières sont consignés dans le tableau II ci-après. Les résultats de l'estimation avec les capacités (colonnes 2 et 3 ) sont globalement significatif à $1 \%$ car avec un Wald chi2(7) $=19,73$, la Prob $>$ chi $2=0,006$. On accepte l'hypothèse de la présence d'un AR (1) des résidus et on ne peut pas rejeter l'absence d'un effet $\mathrm{AR}(2)$ des différents résultats, ceux-ci sont conformes aux hypothèses formulées. Ces résultats montrent qu'à l'exception des autres variables qui sont statistiquement non significatives, la croissance retardée d'une période et l'inflation sont respectivement significatives de $10 \%$ et $1 \%$ et influencent respectivement la croissance du PIB par tête de $0,66 \%$ et $0,42 \%$. Le niveau de la technologie de frontière et l'indicateur des capacités se retrouvent non significatif. Selon l'étude de Aghion et Cohen (2004), à un seuil de 24\% de la technologie de frontière, l'effet des composantes du capital humain change d'influence. Dans cette étude, il est important de déterminer le seuil pour lequel la technologie de frontière pourrait contribuer non seulement à la croissance économique africaine et l'amélioration de l'influence des capacités sur la croissance économique. A un seuil de 0,13 comme valeur de la frontière technologique, les résultats (colonne 4 et 5 ) prouvent que le modèle est globalement significatif à $1 \%$ car avec un Wald chi2 $(7)=59,79$ et la Prob> chi $2=0,000$. On remarque qu'à l'exception de l'inflation et du taux d'IDE, toutes les variables sont significatives. En effet, avec une significativité de 1\%, le degré d'ouverture bonifie la croissance du revenu par tête de 43,18\%, contrairement à la croissance retardée d'une période dont l'effet sur la croissance est de $-0,68 \%$. Les capacités renforcées obtiennent une contribution de $0,85 \%$ à la croissance du PIB par tête.

Tableau II: Résultats de l'estimation par la Méthode des Moments Généralisés

\begin{tabular}{|c|c|c|c|c|c|c|}
\hline \multirow{2}{*}{$\begin{array}{l}\text { Variable à } \\
\text { expliquer } \\
\text { Croissance } \\
\text { du Pib par } \\
\text { tête } \\
\text { Variables }\end{array}$} & \multicolumn{2}{|c|}{$\begin{array}{l}\text { Modèle ICA, Technologie } \\
\text { de frontière } \\
\text { Wald chi2 }(7)=19,73 \\
\text { Prob }>\text { chi } 2=0,006\end{array}$} & \multicolumn{2}{|c|}{$\begin{array}{l}\text { Modèle ICA Technologie de } \\
\text { frontière avec seuil de } 0,13 \\
\text { Wald chi } 2(7)=59,79 \\
\text { Prob> chi } 2=0,000\end{array}$} & \multicolumn{2}{|c|}{$\begin{array}{l}\text { Estimation du ratio-Elève } \\
\text { Pédagogue et la technologie } \\
\text { de frontière } \\
\text { Wald chi2 }(7)=21,57 \\
\text { Prob>chi2 }=0,003\end{array}$} \\
\hline & Val coef (1) & p-value & Val coef (2) & p-value & Val coef & p-value \\
\hline cr_pibt(-1) & 0,166 & $0,069^{*}$ & $-0,682$ & $0,000 * * *$ & 0,032 & 0,678 \\
\hline Tech_fro & 45,11 & 0,553 & 181,11 & $0,040 * *$ & $-706,22$ & 0,107 \\
\hline ICA & $-0,018$ & 0,812 & 0,854 & $0,014 * *$ & & \\
\hline Vin_Intera & $-0,248$ & 0,794 & $-4,643$ & 0,017 & 33,40 & $0,070^{*}$ \\
\hline
\end{tabular}




$\begin{array}{lcccccc}\text { D_ouver } & -0,63 & 0,865 & 43,188 & 0,000 * * * & 18,51 & 0,574 \\ \text { Infla } & 0,42 & 0,001 * * * & -0,083 & 0,724 & 0,48 & 0,008^{* * *} \\ \text { Tx_IDE } & 0,935 & 0,934 & 34,513 & 0,345 & 4,108 & 0,847 \\ \text { R_El_E_se } & & & & & -0,391 & 0,273\end{array}$

Arellano-Bond test for $\mathrm{AR}(1)$ in first differences: $\mathrm{z}=-5.42 \operatorname{Pr}>\mathrm{z}=0.000$

Arellano-Bond test for AR(2) in first differences: $\mathrm{z}=-0.83$ Pr $>\mathrm{z}=0.408$

Arellano-Bond test for AR(1) in first differences: $\mathrm{z}=-2.09 \operatorname{Pr}>\mathrm{z}=0.037$

Arellano-Bond test for $\operatorname{AR}(2)$ in first differences: $z=-1.40 \operatorname{Pr}>z=0.161$

Arellano-Bond test for $\mathrm{AR}(1)$ in first differences: $\mathrm{z}=-4.04 \operatorname{Pr}>\mathrm{z}=0.000$

Arellano-Bond test for $\mathrm{AR}(2)$ in first differences: $\mathrm{z}=-1.07 \operatorname{Pr}>\mathrm{z}=0.283$

Source : auteur, réalisé à partir des résultats obtenus sous Stata $13 . *$ significatif à $10 \%, * *$ significatif à $5 \%$ et $* * *$ significatif à $1 \%$

La technologie de frontière avec ce seuil, permet d'accroître le revenu par tête des pays de l'Afrique subsaharienne à hauteur de $181,11 \%$, mais elle n'encourage pas l'influence du niveau des capacités africaines à la création de valeur économique, à l'antinomie une baisse de 4,64\%. La dernière colonne du tableau concerne le résultat de l'équation sur le capital humain. La significativité globale est justifiée à $1 \%$ avec un Wald chi2(7) $=21,57$. Le retard d'une année de la croissance du revenu par tête, le degré d'ouverture, la technologie de frontière et le ratio de l'IDE n'ont aucun apport contributif à la croissance économique du PIB par tête. Le ratio élève-pédagogue n'a aucune influence importante à l'amélioration de la croissance, mais l'apport de la technologie de frontière permet d'avoir une importante contribution. Avec une significativité de $10 \%$, la technologie de frontière encourage l'influence de l'éducation secondaire (ratio Elève-Enseignant) sur la croissance du PIB par habitant des pays d'Afrique subsaharienne à hauteur de 33,41\%. Ceci suppose que, l'éducation secondaire est appropriée à l'adaptation de la technologie venant d'ailleurs. Ce résultat confirme la thèse selon laquelle loin de la technologie de frontière, le potentiel d'imitation ou d'adaptation est très important pour les pays concernés, ceux-ci ont intérêt à consacrer leurs ressources pour exploiter ce potentiel.

En somme, l'indicateur des capacités renforcées n'a aucun effet sur la croissance du PIB par habitant dans cette situation des pays africains d'être très loin de la technologie de frontière. Mais à partir d'un seuil 0,13 comme 
valeur de la technologie de frontière, l'apport de celle-ci pour l'amélioration de l'influence des capacités renforcées est soldé par une baisse de la croissance du PIB par tête de 4,64\%. A ce même seuil, le niveau actuel des capacités renforcées impacte positivement le PIB par habitant de ces pays de $0,85 \% \%$. Pour ce qui concerne l'éducation secondaire, les résultats confirment aussi que loin de la frontière technologique, elle est appropriée à l'adaptation de la technologie venant ailleurs. Car celle-ci améliore son influence sur la croissance du revenu par habitant des pays de l'Afrique subsaharienne de $33,40 \%$.

\section{Discussion des résultats}

Selon la littérature, les capacités sont censées être source de performance économique (Morgan, 1998, 2006 ; Baser et Morgan, 2008 ; Ksafack, 2011), mais les résultats de l'étude pour le cas des pays africains, montrent qu'elles ne sont pas significatives. En sus, Bien que ces pays d'Afrique Subsaharienne soient loin de la technologie de frontière du monde, leurs capacités renforcées ne sont pas adaptées à la technologie venant d'ailleurs. L'effet d'interaction entre ces deux indicateurs sur la croissance du PIB par tête est négatif. Pour ce qui est de l'éducation secondaire, l'influence négative sur la performance économie des pays d'Afrique Subsaharienne est confirmée par l'étude Acemoglu et Robinson (2008). A cette position éloignée de la technologie de frontière, le niveau de l'éducation est adapté à la technologie venant d'ailleurs. Ce résultat est conforme à ceux développés par Ang et al (2011) et Acemoglu et al (2006) qui confirment que loin de la technologie de frontière, l'éducation primaire et l'éducation secondaire sont appropriées à l'adaptation de la technologie venant d'ailleurs.

\section{Conclusion}

Cette étude a tenté d'analyser l'influence des capacités renforcées et du capital humain sur l'évolution du PIB par habitants de 40 pays de l'Afrique Subsaharienne en tenant compte de leur position à la technologie de frontière du monde. En s'inspirant dans un premier temps, des travaux de Morgan (1998, 2006), Baser et Morgan (2008), et Engel et al (2007) et dans un second, de ceux de Ang et al (2011), Acemoglu et al (2006) et d'Aghion et al (2004), elle donne deux conclusions importantes. La première est que la capacité globale est un indicateur qui crée de la valeur pour le développement économique. La seconde est que l'effet du capital humain (primaire, secondaire et supérieur) sur la croissance économique d'un pays dépend de sa position à la technologie de frontière mondiale.

L'analyse empirique a été possible grâce à la méthode des moments généralisés. Les résultats prouvent que les capacités renforcées des pays de l'Afrique subsaharienne n'ont aucune influence sur l'accroissement du PIB 
par habitant. La technologie de frontière n'a aucun apport contributif à l'accroissement du revenu par habitant, son apport à l'amélioration de l'incidence des capacités renforcées sur l'évolution du PIB par habitant est sans effet. Toutefois, à un seuil de 0,13 comme valeur de la technologie de frontière des pays de l'Afrique subsaharienne, le revenu par habitant est bonifié de $181,11 \%$ par celle-ci, mais sa contribution à l'effet des capacités africaines est négative $(-4,64 \%)$. Les résultats montrent également que le capital humain est non significatif. A travers l'effet d'interaction, la technologie de frontière permet de hausser l'effet du capital humain sur le PIB par habitant à hauteur de 33, 40\%. Ainsi, l'éducation secondaire des pays de l'Afrique subsaharienne est appropriée à l'adaptation des technologies venant d'ailleurs. Pour que l'Afrique subsaharienne parvienne à accroître et maintenir sa croissance économique sur une longue période, il lui faut : veiller à un meilleur usage des ressources affectées au système éducatif. Améliorer le ratio élève-enseignant du niveau d'éducation secondaire. Accroître également l'investissement dans l'amélioration de la capacité de la main d'œuvre capable d'être adaptée à la technologie venant des pays développés. Améliorer le niveau des capacités, en augmentant le budget affecté au processus des capacités. Toutefois, les gouvernements africains doivent ensemble conjuguer leurs efforts pour créer les environnements favorables à l'égard des politiques favorisant l'accroissement du niveau de technologie de frontière.

\section{References:}

1. ACBF. (African Capacity Building Foundation). (2011). Africa Capacity Indicators : Capacity Development in Fragile States. Harare, Zimbabwe

2. ACBF. (The African Capacity Building Foundation) (2015). Africa Capacity Report 2015: Capacity Imperatives for Domestic Resource Mobilization in Africa. Harare, Zimbabwe

3. Acemoglu, D., Aghion, P,. \& Zilibotti, F. (2006). Distance to frontier, selection, and economic growth. Journal of the European Economic Association, vol 4, $\mathrm{N}^{\circ}$ 1, pp. 37-74. https://doi.org/10.1162/jeea.2006.4.1.37

4. Acemoglu, D., \& Robinson, J. (2010). The role of institutions in growth and developments. Review of Economics and Institutions, Vol. 1, $\mathrm{N}^{\circ} 2$ Article 1. https://doi.org/10.5202/rei.v1i2.1

5. Aghion, P., Askenazy P., Bourlès R., Cette, G., \& Dromel, N. (2008). Distance à la frontière technologique, rigidités de marché, éducation et croissance. Économie et statistique, $\mathrm{N}^{\mathrm{o}}$ 419-420, pp. 1130. https://doi.org/10.3406/estat.2008.7725

6. Aghion, P., Bloom, N., Blundell, R., Griffith, R., \& Howitt, P. (2005). Competition and Innovation: An Inverted-U Relationship. The 
Quarterly Journal of Economics. Oxford University, Vol. 120, $\mathrm{N}^{\circ} 2$, pp. 701-728. https://doi.org/10.1093/qje/120.2.701

7. Aghion, P., \& Cohen, É. (2004). Éducation et croissance. La documentation française. Paris, ISBN:2-11-05533-2

8. Altinok, N. (2007). Capital humain et croissance : l'apport des enquêtes internationales sur les acquis des élèves. Économie publique, Vol. 18-19, $\mathrm{N}^{\circ}$ 2-1, https://doi.org/10.4000/economiepublique.4762

9. Ang, J., B., Madsen, J., B., \& Islam, M., D. (2011). The effets of human capital composition on technological convergence. Elsevier, journal of macroeconomics. Vol 33, $\mathrm{N}^{0}$ 3, pp. 465-476. https://doi.org/10.1016/j.jmacro.2011.03.001

10. Angel, F., \& Antonio, C. (2002). Le capital humain dans une économie mondiale fondée sur la croissance. Rapport final Luxembourg: Office des publications officielles des Communautés européennes 2003-VI.

11. Banerjee, R., \& Sinha, S., R. (2014). Human capital, technological progress and trade: What explains India's long run growth?. Journal of Asian Economics, Vol 30, pp. 15-31. https://doi.org/10.1016/j.asieco.2013.12.003

12. Barro, R., J. (2001). Human capital and growth. The American $\begin{array}{llllll}\text { Economic } & \text { Review, Vol. } 91 & \mathrm{~N}^{\circ} & 2, & \text { pp. } & 12-17 .\end{array}$ https://doi.org/10.1257/aer.91.2.12

13. Baser, H., \& Morgan, P. (2008). Capacity, change and performance study report. Discussion Paper $N^{0} 59$ B. European Centre for Development Policy Management

14. Benhabib, J., \& Spiegel, M. (1994). The role of human capital in economic development: evidence from aggregate cross-country data. Journal of Monetary Economies, Vol. 34, $\mathrm{N}^{\circ} 2$, pp. 143-174. https://doi.org/10.1016/0304-3932(94)90047-7

15. Bils, M., \& Klenow, P. (2000). Does schooling cause growth", American economic Review, Vol. 90, $\mathrm{N}^{0} 5$, pp. 1160-1183. https://doi.org/10.1257/aer.90.5.1160

16. Denison, E., F. (1962). The sources of economic growth in the United States and the alternatives before us. Committee for Economic Development. Supplementary Paper 13. New York : Committee for Economic Development. ch. 7, pp. 77 - 80.

17. Engel, P., Keijzer, N., \& Land, T. (2007). A balanced approach to monitoring and evaluating capacity and performance. Discussion paper No 58E, European Centre for Development Policy Management.

18. Gerschenkron, A. (1962). Economie Backwardness in Historical Perspective : A book of essays. Belknap Press of Harvard University Press. 
19. Hall, R., E., \& Jones, C., I. (1999). Why Do Some Countries Produce So Much More Output Per Worker Than Others?. The Quarterly Journal of Economics, Vol. 114, No. 1 pp. 83-116. https://www.jstor.org/stable/2586948

20. Hanushek, E., .A., \& Kimko, D., D. (2000). Schooling, Labor-Force Quality, and the Growth of Nations. American Economic Review, Vol. 90, No 5, pp. 1184-1208. https://doi.org/10.1257/aer.90.5.1184

21. Islam, R. (2010). Human Capital Composition, Proximity to Technology Frontier and Productivity Growth. Department of economics, discussion paper 23/10. Pp. 1-53.

22. Organisation De Coopération Et De Développement Économiques OCDE. (2006). Relever le défi posé par le renforcement des capacités : Evoluer vers la bonne pratique.

23. Pritchett, L. (2000). Where has all the education gone? World Bank and Kennedy School of Government Revised.

24. Mahshi, K. (2006). Développement des capacités dans l'éducation. Lettre d'information de l'IIPE de l'UNESCO, vol. XXIV, $\mathrm{n}^{\circ} 4$.

25. Morgan, P. (2006). The concept of capacity. European Centre for Development Policy Management.

26. North, D., C., William, S., \& Barry, W. (1999). Order, Disorder and Economic Change: Latin America vs North America. Unpublished Manuscript, Hoover Institution, Stanford University.

27. Rapport (PNUD). (2008). Le développement des capacités. Octobre, note de pratique PNUD 304 east 45 th street New york, ny 10017 (etats-unis)

28. Reboud, V. (2008). Amartya Sen : un économiste du développement? Agence Française de Développement.

29. Romer, P., M. (1991). Progrès technique endogène. Annales d'Economie et de statistique $\mathrm{N}^{\mathrm{o}}$ 22, pp.1-32. https://doi.org/10.2307/20075820

30. Romer, P., M. (1990). Endogenous Technological Change. Journal of Political Economy, Vol. 98, No. 5, Part 2: The Problem of Development: A Conference of the Institute for the Study of Free Enterprise Systems (Oct., 1990), pp. S71-S102.

31. Schultz, T., W. (1972). Human Capital: Policy Issues and Research Opportunities. Vol. 6, pp.1-84.

32. Ksafack, N., R. (2011). L'état du renforcement des capacités en Afrique. Accasionalpaper $\mathrm{N}^{\mathrm{o}} 13$. Fondation pour le Renforcement des Capacités en Afrique.

33. Ubels, J., Aku, N., Baddoo, A. \& Fowler, A. (2010). Capacity Development in Practice. Development Organisation.89832478. 
34. Vandenbussche, J., Aghion, P. \& Meghir, C. (2006). Growth, distance to frontier and composition of human capital. Journal of Economic growth, $\mathrm{N}^{\mathrm{o}} 11$, pp. 97-127. https://doi.org/10.1007/s10887-006-9002$\mathrm{y}$

35. Wooldridge, M. J. (2004). Introductory econometric: a modern

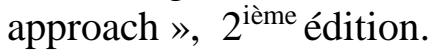

